\title{
CALCITONIN GENE-RELATED PEPTIDE IN SERUM AFTER SPONTANEOUS SUBARACHNOID HEMORRHAGE
}

\author{
Karl-Michael Schebesch, Elisabeth Bruendl Andreas Hochreiter, \\ Judith Scheitzach, Sylvia Bele Andreas Herbst, Alexander Brawanski, \\ Martin Proescholdt, Anette Lohmeier, Eva-Maria Stoerr and Petra Schoedel \\ Department of Neurosurgery, University Medical Center Regensburg, \\ Franz-Josef-Strauss Allee 11, 93053 Regensburg, Germany
}

Received 2014-03-04; Revised 2014-06-16; Accepted 2014-06-28

\begin{abstract}
Endogenous Calcitonin Gene-Related Peptide (CGRP) stored in perivascular nerve fibers around the cerebral arteries mediates vasodilation and regulates cerebral blood flow under physiological conditions. Increased levels of CGRP have been continually reported in patients with migraine, but the role of CGRP in the pathophysiology of Subarachnoid Hemorrhage (SAH) has not yet been sufficiently evaluated. Over 10 days, serum was prospectively collected from 33 consecutive patients (17 women, 16 men; mean age 52.8 years) with spontaneous non-traumatic SAH. All patients were graded III or IV according to the Fisher Score. CGRP levels were determined by means of an Enzyme-Immunosorbent Assay (EIA). CGRP concentrations were correlated to (1) the anatomical localization of the aneurysm (anterior circulation or posterior circulation), (2) the treatment modality (clipped group, coiled group, or untreated group) and (3) the rate of ischemia determined by computed tomography (ischemia group, non-ischemia group, or iatrogenic ischemia group). (1) Anatomical localization: CGRP levels were significantly higher in patients with a ruptured aneurysm of the anterior circulation $(\mathrm{p}<0.001)$. (2) Treatment modality: CGRP levels differed significantly between the groups (coil group, clip group, or untreated group; $\mathrm{p}<0.001$ ). The highest levels of CGRP were detected in the coil group and the lowest levels in the untreated group. About (3) Ischemia: CGRP levels differed significantly between the three groups (ischemia group, non-ischemia group, or iatrogenic ischemia group; $\mathrm{p}=0.038$ ). The highest levels of CGRP were found in the ischemia group. The maximum levels of CGRP in serum were found for patients with a ruptured aneurysm of the anterior circulation and subsequent ischemia treated by endovascular coiling. These findings support the theory that CGRP is produced and excessively released to counteract cerebral vasospasm and subsequent ischemia. Presumably, the serum levels of CGRP are affected by the anatomical localization of the aneurysm and can be stimulated by any endoluminal manipulation of the parent artery.
\end{abstract}

Keywords: Neuropeptides, Calcitonin Gene-Related Peptide, Cgrp, Subarachnoid Hemorrhage, Biomarker, Outcome, Cerebral Ischemia, Vasospasm

\section{INTRODUCTION}

Although the peptidergic regulation of the cerebral vasculature under normal conditions is well established (Edvinsson et al., 1994; McCulloch et al., 1986; Hokfelt et al., 1992), its role in pathophysiology after spontaneous Subarachnoid Hemorrhage (SAH), arterial vasospasm and Cerebral Ischemia (CI) remains unclear (Kokkoris et al., 2012). Recently, Jung et al. (2013) have emphasized the importance of endogenous molecular and peptidergic biomarkers that potentially predict cerebral vasospasm, subsequent $\mathrm{CI}$ and functional outcome after SAH and traumatic brain injury.

Corresponding Author: Karl-Michael Schebesch, Department of Neurosurgery, University Medical Center Regensburg, Franz-Josef-Strauss Allee 11, 93053 Regensburg, Germany Tel: ++49 9419449010 Fax: ++49 9419449002 
Besides biomarkers for inflammation, biomarkers for oxidative stress and vascular biomarkers, neuropeptides have been a subject of intensive research over the past few decades. Of particular interest was the potent vasoconstrictor Neuropeptide Y (NPY) and its possible role in the pathophysiology of vasospasm-induced CI due to SAH (Heilig et al., 1990; Pluta et al., 1992; Schebesch et al., 2013a). One of the most important functional antagonists of NPY is CGRP. Described the functional antagonism of NPY and the vasodilator Calcitonin Gene-Related Peptide (CGRP) in mesenteric resistance arteries (De Mey et al., 2008). This antagonism had also been proposed to be present in the central nervous system and some research groups have evaluated the possible role of CGRP in SAH (Kokkoris et al., 2012; Juul et al., 1990; 1995; Schebesch et al., 2013b).

CGRP is a 37 -amino-acid neuropeptide. The signal transduction is obtained by G-protein-coupled receptors, thereby activating adenylate cyclase (Rosenfeld et al., 1983). CGRP is expressed and stored in nervous tissue, such as the sensory and the trigeminal ganglia, the cortex and the pituitary gland. Cerebral perivascular fibers containing CGRP in presynaptic vesicles almost exclusively derive from the Gasserian ganglion (McCulloch et al., 1986; Hokfelt et al., 1992; Saria et al., 1992). CGRP physiologically restores the vascular tone after vasoconstriction and represents the most important mediator of the 'trigeminovascular reflex'. The vascular tone is increased by the intrinsic release of vasoconstrictive neuropeptides, such as NPY, Vasointestinal Peptide (VIP) and Substance P (SP). Thus, Cerebral Blood Flow (CBF) is restrained, whereas CGRP release relaxes the smooth muscle layer, dilates the artery and increases the CBF volume (Johnston et al., 1990).

Excessive CGRP release has been identified as one of the main causes of migraine because CGRP unalterably dilates the cerebral vessels (Edvinsson et al., 1994). The role of CGRP in the pathophysiology of vasospasmrelated CI due to $\mathrm{SAH}$ and its potential in preventing SAH-related stroke have been advocated before (Kokkoris et al., 2012; Johnston et al., 1992; 1990). However, to the best of our knowledge, no data are yet available on the influence of the anatomical localization of the ruptured aneurysm and the effect of endovascular or surgical occlusion on the intrinsic release and dynamics of CGRP in serum.

\section{PATIENTS AND METHOD}

This study included 33 consecutive patients (17 women, 16 men) with spontaneous non-traumatic SAH. Their mean age was 52.8 years, ranging from 28 years to 81 years. All patients presented with sudden onset of SAH symptoms and were admitted to our neurosurgical Intensive Care Unit (ICU) within $12 \mathrm{~h}$. Each patient received an External Ventricular Drainage (EVD) in combination with an Intraventricular Intracranial Pressure (ICP) device (RAUMEDIC, Rehau, Germany) within $24 \mathrm{~h}$. Continuous ICP monitoring was installed. Exclusion criteria were presence of any immune-modulatory or chronic inflammatory diseases, history of repeated $\mathrm{SAH}$, or SAH induced by trauma.

The volume of subarachnoid blood was screened on the initial CT scan and classified according to the score established by (Fisher et al., 1980) (Fisher Score, Table 1). All patients were classified with a Fisher score of III or IV $(n=22$ and $n=11$ respectively, Table 2). Each patient underwent early digital subtraction angiography (DSA, $<24 \mathrm{~h}$ after admission). About 29 ruptured aneurysms were identified, Table 3. Aneurysmal occlusion was achieved by endovascular Coiling (Coil) in 22 patients and by surgical Clipping (Clip) in 6 patients within 48 $\mathrm{h}$ after the onset of SAH. Within $24 \mathrm{~h}$ after surgery or intervention, another CT was conducted to rule out or confirm procedure-related ischemia.

All patients were treated according to our ICU standard operating protocol (Kagerbauer et al., 2010). Basically, Transcranial Doppler sonography (TCD) examinations were conducted daily. The occurrence of vasospasm was suspected if the mean blood flow velocity in the MCA or ACA was higher than $150 \mathrm{~cm}$ $\sec ^{-1}$ (Woitzik et al., 2012). Patients were examined under sedation via a CT scan if the occurrence of vasospasm was suspected by TCD and if the ICP level was above $20 \mathrm{~mm} \mathrm{Hg}$ and permanently refractory to the administration of osmotic agents or to the application of additional sedatives for at least $30 \mathrm{~min}$.

Every morning between 8 and $12 \mathrm{am}, 5 \mathrm{~mL}$ of arterial blood were drawn directly from the arterial catheter (radial artery). The observational period was 10 days after the onset of SAH. Subgroups were formed according to the localization of the ruptured aneurysm, the treatment modality and the development of vasospasm-related $\mathrm{CI}$ during the first 10 days. 
Table 1. Fisher score

\begin{tabular}{ll}
\hline I & No hemorrhage evident \\
\hline II & Subarachnoid hemorrhage measuring less than $1 \mathrm{~mm}$ \\
III & Subarachnoid hemorrhage measuring more than $1 \mathrm{~mm}$ \\
IV & Subarachnoid hemorrhage of any thickness with intra-ventricular hemorrhage or parenchymal extension \\
\hline
\end{tabular}

Table 2. Descriptive data $(\mathrm{N}=33)$

\begin{tabular}{lc}
\hline Ratio men (\%)/women (\%) & $16(48.5) / 17(51.5)$ \\
\hline Age (yrs.) & 52.8 \\
Mean & 52.0 \\
Median & 28 to 81 \\
Range & $24(72.5)$ \\
Aneurysmal Localization & $5(15)$ \\
Anterior circulation (\%) & $4(12.5)$ \\
Posterior circulation (\%) & \\
No aneurysm detected (\%) & $6(18.5)$ \\
Treatment of Aneurysms & $22(66.5)$ \\
Clip (\%) & $5(15)$ \\
Coil (\%) & \\
No treatment (\%) & $10(30.5)$ \\
Cerebral Ischemia (CI) & \\
Unrelated to manipulation (\%) & $5(15)$ \\
Related to manipulation (\%) & $18(54.5)$ \\
No CI & \\
Fisher Grade & $22(66.5)$ \\
Grade III (\%) & $11(33.5)$ \\
Grade IV (\%) & \\
\hline
\end{tabular}

Table 3. Anatomical localization of the aneurysmAnterior circulation

\begin{tabular}{lr}
\hline Anterior circulation & $\mathrm{n}$ \\
\hline Middle Cerebral Artery (MCA) & 5 \\
Internal Carotid Artery (ICA) & 3 \\
Anterior communicating artery (Acomm) & 14 \\
Pericallosal artery & 1 \\
Posterior Cerebral Artery (PCA) & 1 \\
Posterior circulation & 2 \\
Basilar Artery (BA) & 2 \\
Vertebral Artery (VA) & 1 \\
Posterior communicating artery (Pcomm) & 4 \\
No aneurysm & \\
\hline
\end{tabular}

The study protocol was approved by the local ethics committee (06/179).

\subsection{Laboratory Procedures}

Immediately after sampling, serum samples were centrifuged at 1200 rounds per minute (rpm) for 10 min. The supernatants were aliquoted and stored at$80^{\circ}$ until further use. The samples were thawed, aliquoted $(1.5 \mathrm{~mL})$ with columns (STRATA C18-E (55 $\mu \mathrm{m}, 70 \mathrm{~A}) 200 \mathrm{mg} / 3 \mathrm{ml}$-columns, Phoenix
Pharmaceuticals Inc., Burlingame, USA), purified, evaporated on a vacuum concentrator and dissolved in $250 \mu \mathrm{L}$ assay-buffer, which resulted in a six times higher concentration. CGRP levels were measured in duplicate in purified serum using competitive Enzyme Immunoassay (EIA).

\subsection{Statistical Analysis}

All data are expressed as the mean value plus the Standard Error of the Mean (SEM). Different groups were compared with the One Way Analysis of Variance (ANOVA) with all pairwise multiple comparison procedure (Holm-Sidak Method; Sigma Stat Version 3.0, SPSS Inc., Chicago, IL, USA). The level of significance was set at $\mathrm{p}<0.05$.

\section{RESULTS}

About 24 patients had a ruptured anterior circulation aneurysm and 5 patients had a ruptured posterior circulation aneurysm. In 4 patients, no aneurysm was detected angiographically. For details Table 3. 
Aneurysmal occlusion was neither possible in the 4 patients with non-aneurysmal SAH nor in 1 female patient with a right ICA aneurysm, who did not wish any further treatment.

About 22 patients were treated with endovascular occlusion and 6 patients with surgical clipping. No rebleeding or intra-procedural re-ruptures occurred.

About 10 patients developed 17 vasospasm-related ischemic lesions in the anterior circulation territories: $\mathrm{n}=$ 2 in the right Middle Cerebral Artery (MCA), $n=5$ in the left MCA, $n=4$ in the right Anterior Cerebral Artery (ACA) and $n=6$ in the left ACA. 18 patients had no ischemia, but 5 patients developed cerebral ischemia due to endovascular treatment $(\mathrm{n}=1)$ or surgical clipping ( $\mathrm{n}$ $=4$ ). These 5 patients were outlined as an iatrogenic ischemia group:

- CGRP levels according to the anatomical localization of the aneurysm, Fig. 3

- CGRP levels were significantly higher in patients with a ruptured aneurysm of the anterior circulation $(p<0.001)$ The highest levels of CGRP were detected on day 4,5 and 7

- In the beginning, the CGRP levels in the anterior circulation increased, reaching the maximum on day 4 and then steadily decreased. The CGRP levels of the posterior circulation group and the no-aneurysm group steadily increased over the observational period

\subsection{CGRP Levels According to the Treatment Modality, Fig. 1:}

- CGRP levels differed significantly between the groups (coil group, clip group and untreated group; $\mathrm{p}<0.001)$. The highest levels of CGRP were detected in the coil group and the lowest levels in the untreated group

- In the coil group, CGRP levels were significantly increased on day 3, 4 and 5 after the onset of SAH. CGRP levels in the clip group and in the untreated group did not vary significantly over the observational period

\subsection{CGRP Levels According to Vasospasm- Related Ischemia, Fig. 2:}

- CGRP levels differed significantly between the three groups (ischemia, no ischemia, iatrogenic ischemia; $\mathrm{p}=0.038)$. The highest levels of CGRP were found in the ischemia group

- In the ischemia group, CGRP levels increased steadily from day 1 to day 4 and then decreased again. CGRP levels in the no ischemia group and in the iatrogenic ischemia group showed no significant changes over the observational period

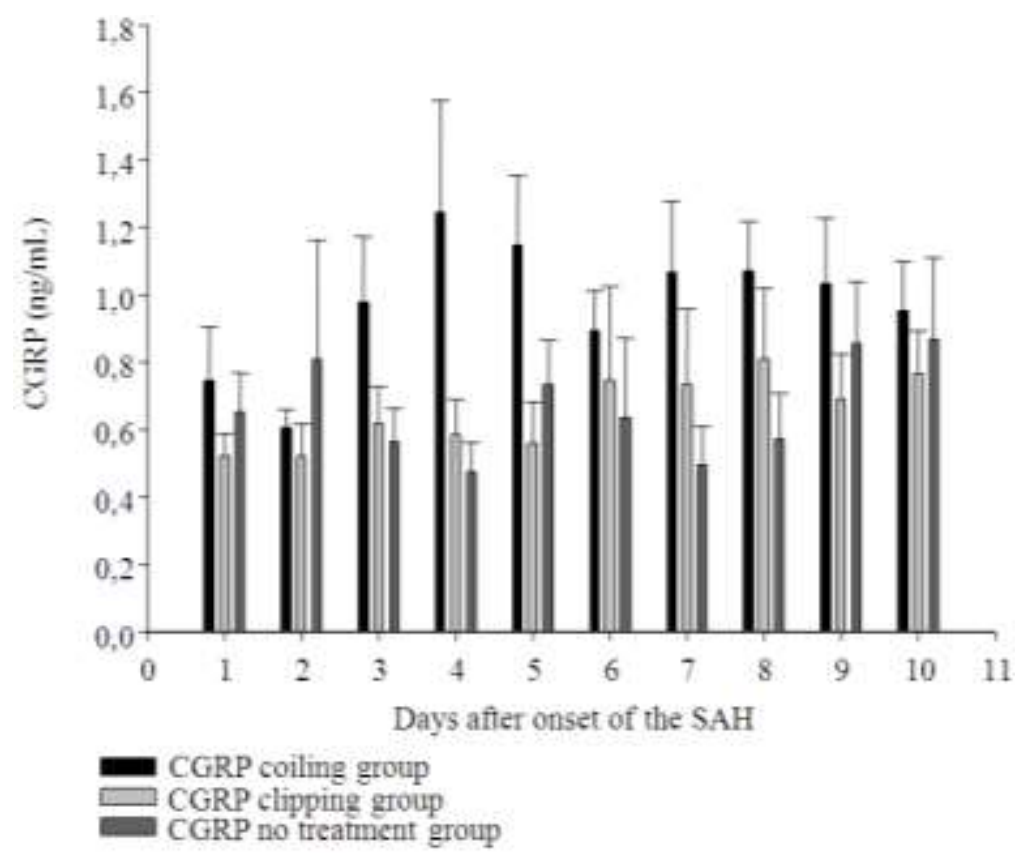

Fig. 1. CGRP levels according to the treatment modality 


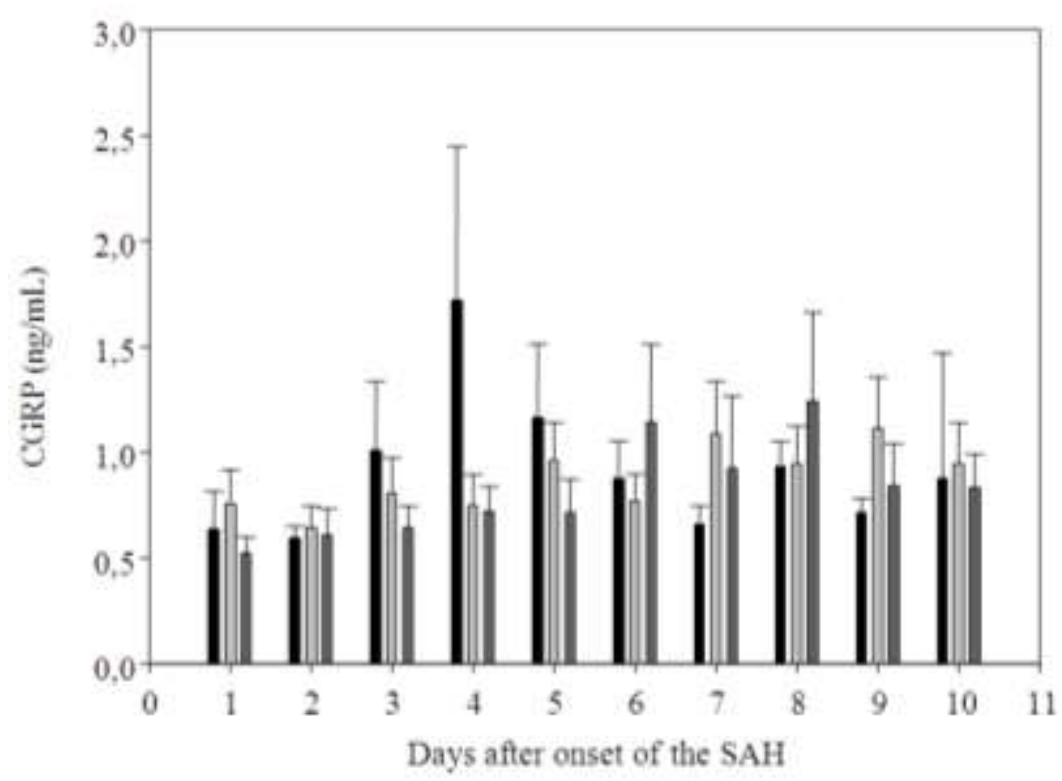

CGRP ischemia group

$\square$ CGRP no ischemia group

CGRP iatrogenic ischemia group

Fig. 2. CGRP levels according to ischemia
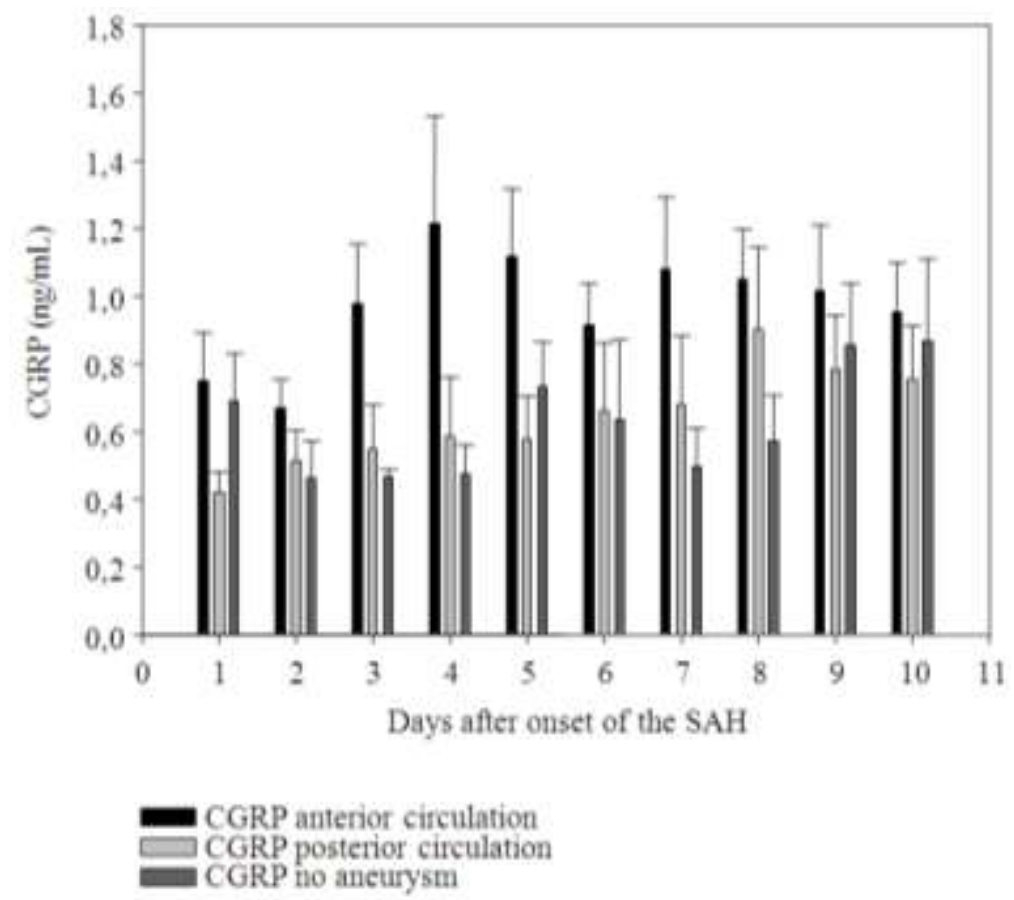

Fig. 3. CGRP levels according to the anatomical localization of the aneurysm 


\section{DISCUSSION}

CGRP is one of the most potent endogenous vasodilators in humans (Hokfelt et al., 1992; Kokkoris et al., 2012). Intrinsic production in the trigeminal ganglion of Gasseri, neuronal transport, presynaptic release and consecutive re-uptake have been evaluated in detail and the major role of CGRP in migraine has been repeatedly confirmed (Guldiken et al., 2013). However, consistent data on CGRP in the context of aneurysmal SAH-related vasospasm and subsequent $\mathrm{CI}$ are still missing.

In early SAH research, the trigemino-vascular reflex-mainly modulated by the intrinsic neuropeptide CGRP-had been investigated several times. Finally Edvinsson et al. (1987) could confirm that, in cats, the presence of lesions in the trigeminal ganglion prolonged arterial vasoconstriction after SAH (McCulloch et al., 1986). However, six years later, Keller et al. (1993) studied the profile of neuropeptides in the dura mater in a singlehemorrhage rodent model. In this study, the profile of CGRP remained unchanged in contrast to intensive, SAH-related immunostaining for NPY. The authors concluded that the endovascular immunoreactivity corresponded variably to different stimuli and that CGRP seemed to be unaffected by SAH (Keller et al., 1993). When determining the level of arterial CGRP in SAH patients in our clinical series, we could show that the levels of CGRP in serum correlated to the anatomical localization of the ruptured aneurysm. We interpret the discrepancy between Keller's results and our data as another indication for the poor comparability between artificial SAH in animal studies and real SAH after the rupture of an aneurysm in a clinical setting. In other words, we believe that the excessive release of neuropeptides stored in perivascular nerve fibers could be caused by the direct affection or disruption of the nerve fibers at the site of the ruptured aneurysm. Whether the development of an aneurysm and subsequent vascular remodeling induces higher compaction of synaptic vesicles of perivascular nerves should be evaluated in future studies.

Juul et al. (1995) considered CGRP as an important marker for ischemic stroke after SAH. CGRP in serum was significantly increased in a study population of 20 patients with SAH, severe vasospasm and marked hemodynamic changes. Similarly, we found the highest CGRP level in the vasospasm-related ischemia group compared to the iatrogenic ischemia group and the group without ischemia. Furthermore, the course of the serum levels of CGRP in our population was equal to the course of CGRP described by Juul et al. (1995) who documented the highest levels of CGRP in MCA aneurysms. This finding was also supported by our data.

The therapeutic value of CGRP after SAH was in the focus of interest in the early 1990s. Conducted a randomized multicenter study that included 15 patients with neurological deficits. The patients in the CGRPinfusion group improved significantly (Johnston et al., 1990). Consequently, the 'European CGRP in SAH study $^{6}$ was started in 1992 including 117 patients of whom 62 received CGRP-infusions (Johnston et al., 1992). Because the patients showed severe arterial hypotension, the study was prematurely terminated, so that no statistical analysis was conducted. Nevertheless, in the elaborated review of the literature concerning the role of CGRP in SAH, Kokkoris et al. (2012) emphasized the need for more research into the contribution of CGRP in SAH.

In contrast to NPY-that was significantly increased in both serum and CSF in almost equal proportion (Schebesch et al., 2013a; Suzuki et al., 1989; Uemura et al., 1987), the levels of CGRP in serum were inversely proportional to the levels of CGRP in Cerebrospinal Fluid (CSF). The levels of CGRP in CSF had been examined before by Juul et al. (1995). This study showed that CGRP levels in CSF were markedly increased whereas the corresponding CGRP levels of a control group were not measurable. Likewise, we could show that CGRP was excessively released into CSF in 12 patients after aneurismal SAH compared to a control group (Schebesch et al., 2013b). During the first four days, the CGRP levels in CSF of the vasospasm group ranged significantly lower than the respective levels in the nonvasospasm group. In contrast, the relationship between CGRP and vasospasm-associated ischemia in serum seems to be inversed. In serum, high levels of CGRP were correlated to a higher rate of cerebral ischemia. This finding corroborates the results of Juul et al. (1995) who found high serum levels and low CSF levels of CGRP in patients with vasospasm after SAH.

We have no valid explanation for the finding that significantly higher levels of CGRP were detected in the coil group than in the clip group and the untreated group. Presumably, endoluminal mechanical manipulation of the aneurysm-bearing artery resulted in the excessive release of CGRP and thus in increased serum levels. However, this result would support the hypothesis that the development of an aneurysm induces higher 
compaction of perivascular nerves that leads to the excessive release of neuropeptides in case of mechanical stimulation of the parent vessel.

\subsection{Limitations}

This study has several limitations. First, as usual in prospective observational studies involving the inclusion of consecutive patients, the numbers of patients in the subgroups are dissimilar. Second, our study does not quantify CGRP and serum in the context of, for example, stroke volume, aneurysm diameter and shape, clinical course and profile of the medical therapy and nutrition during the ICU stay. These issues should be addressed in further prospective research projects. Third, no control group with normal values of CGRP in serum was included.

\section{CONCLUSION}

Despite certain limitations we conclude that the serum levels of CGRP after SAH depend on the anatomical localization of the ruptured aneurysm. Mechanical manipulation of the parent vesselparticularly in terms of endovascular coiling-may additionally influence the intrinsic release of CGRP. Further studies should be conducted to investigate whether CGRP is a suitable biomarker for cerebral ischemia due to aneurismal SAH.

\subsection{Disclosure}

The authors declare no conflict of interest.

\section{REFERENCES}

De Mey, J.G., R. Megens and G.E. Fazzi, 2008. Functional antagonism between endogenous neuropeptide $\mathrm{Y}$ and calcitonin gene-related peptide in mesenteric resistance arteries. J. Pharmacol Exp. Ther., 324: 930-7. DOI: 10.1124/jpet.107.133660

Edvinsson, L., I. Jansen, M. Cunha e Sa and S. Gulbenkian, 1994. Demonstration of neuropeptide containing nerves and vasomotor responses to perivascular peptides in human cerebral arteries. Cephalalgia, 14: 88-96.

DOI: 10.1046/j.1468-2982.1994.1402088.x

Edvinsson, L., R. Ekman, I. Jansen, J. McCulloch and R. Uddman, 1987. Calcitonin gene-related peptide and cerebral blood vessels: Distribution and vasomotor effects. J. Cereb. Blood Flow Metab., 7: 720-8. DOI: $10.1038 /$ jcbfm.1987.126
Fisher, C.M., J.P. Kistler and J.M. Davis, 1980. Relation of cerebral vasospasm to subarachnoid hemorrhage visualized by computerized tomographic scanning. Neurosurgery, 6: 1-9. DOI: $10.1227 / 00006123-198001000-00001$

Guldiken, B., T. Sipahi, R. Tekinarslan, L. Kabayel and H. Ozkan et al., 2013. Calcitonin gene related peptide gene polymorphism in migraine patients. Can J. Neurol. Sci., 40: 722-5. PMID: 23968948

Heilig, M., E. Widerlov and Y. Neuropeptide, 1990. An overview of central distribution, functional aspects and possible involvement in neuropsychiatric illnesses. Acta Psychiatr Scand, 82: 95-114. DOI: 10.1111/j.1600-0447.1990.tb01366.x

Hokfelt, T., U. Arvidsson, S. Ceccatelli, R. Cortes and S. Cullheim et al., 1992. Calcitonin gene-related peptide in the brain, spinal cord and some peripheral systems. Ann. N Y Acad. Sci., 657: 119-34. DOI: 10.1111/j.1749-6632.1992.tb22762.X

Johnston, F.G., B.A. Bell, I.J. Robertson, J.D. Miller and C. Haliburn et al., 1990. Effect of calcitonin-generelated peptide on postoperative neurological deficits after subarachnoid haemorrhage. Lancet, 335: 869-72. DOI: 10.1016/0140-6736(90)90473-I

Johnston, F.G., B.A. Bell, I.J. Robertson, J.D. Miller and C. Haliburn et al., 1992. Effect of calcitonin-generelated peptide in patients with delayed postoperative cerebral ischaemia after aneurysmal subarachnoid haemorrhage. Eur. CGRP Subarachnoid Haemorrhage Study Group. Lancet, 339: 831-834. PMID: 1347857

Jung, C.S., B. Lange, M. Zimmermann and V.C.S.F. Seifert, 2013. Serum biomarkers focusing on cerebral vasospasm and ischemia after subarachnoid hemorrhage. Stroke Res. Treat, 2013: 560305.

Juul, R., H. Hara, S.E. Gisvold, A.O. Brubakk and T.A. Fredriksen et al., 1995. Alterations in perivascular dilatory neuropeptides (CGRP, SP, VIP) in the external jugular vein and in the cerebrospinal fluid following subarachnoid haemorrhage in man. Acta Neurochir (Wien), 132: 32-41. DOI: 10.1007/BF01404845

Juul, R., L. Edvinsson, T.A. Fredriksen, R. Ekman and A.O. Brubakk et al., 1990. Changes in the levels of neuropeptide Y-LI in the external jugular vein in connection with vasoconstriction following subarachnoid haemorrhage in man. Involvement of sympathetic neuropeptide $\mathrm{Y}$ in cerebral vasospasm. Acta Neurochir (Wien), 107: 75-81. DOI: $10.1007 / \mathrm{BF} 01405783$ 
Kagerbauer, S.M., D.M. Kemptner, C.P. Schepp, S. Bele and R.D. Rothorl et al., 2010. Elevated premorbid body mass index is not associated with poor neurological outcome in the subacute state after aneurysmal subarachnoid hemorrhage. Cent Eur. Neurosurg., 71: 163-6.

DOI: $10.1055 / \mathrm{s}-0030-1249043$

Keller, J.T., B.G. Mullen and M. Zuccarello, 1993. Dural neuropeptide changes after subarachnoid hemorrhage in rats. Brain Res. Bull., 31: 713-8. DOI: 10.1016/0361-9230(93)90146-3

Kokkoris, S., P. Andrews and D.J. Webb, 2012. Role of calcitonin gene-related peptide in cerebral vasospasm and as a therapeutic approach to subarachnoid hemorrhage. Front Endocrinol. (Lausanne), 3: 135-135.

DOI: 10.3389/fendo.2012.00135, PMID: 23162536

McCulloch, J., R. Uddman, T.A. Kingman and L. Edvinsson, 1986. Calcitonin gene-related peptide: Functional role in cerebrovascular regulation. Proc. Nat. Acad. Sci. USA, 83: 5731-5.

DOI: 10.1073/pnas.83.15.5731

Pluta, R.M., A. Deka-Starosta, A. Zauner, J.K. Morgan and K.M. Muraszko et al., 1992. Neuropeptide Y in the primate model of subarachnoid hemorrhage. J. Neurosurg, 77: 417-23.

DOI: $10.3171 /$ jns.1992.77.3.0417

Rosenfeld, M.G., J.J. Mermod, S.G. Amara, L.W. Swanson and P.E. Sawchenko et al., 1983. Production of a novel neuropeptide encoded by the calcitonin gene via tissue-specific RNA processing. Nature, 304: 129-35.

DOI: $10.1038 / 304129 \mathrm{a} 0$
Saria, A., G. Bernatzky, C. Humpel, C. Haring and G. Skofitsch, 1992. Calcitonin gene-related peptide in the brain. Neurochemical and behavioral investigations. Ann. N Y Acad. Sci., 657: 164-9. DOI: 10.1111/j.1749-6632.1992.tb22765.x

Schebesch, K.M., A. Brawanski, S. Bele, P. Schodel and A. Herbst et al., 2013a. Neuropeptide Y-an early biomarker for cerebral vasospasm after aneurysmal subarachnoid hemorrhage. Neurol. Res., 35: 1038-43. DOI: 10.1179/1743132813Y.0000000246

Schebesch, K.M., A. Herbst, S. Bele, P. Schodel and A. Brawanski et al., 2013b. Calcitonin-gene related peptide and cerebral vasospasm. J. Clin. Neurosci., 20: 584-6. DOI: 10.1016/j.jocn.2012.07.006

Suzuki, Y., S. Sato, H. Suzuki, J. Namba and R. Ohtake et al., 1989. Increased neuropeptide Y concentrations in cerebrospinal fluid from patients with aneurysmal subarachnoid hemorrhage. Stroke, 20: 1680-4. DOI: 10.1161/01.STR.20.12.1680

Uemura, Y., T. Sugimoto, S. Okamoto, H. Handa and N. Mizuno, 1987. Changes of neuropeptide immunoreactivity in cerebrovascular nerve fibers after experimentally produced SAH. Immunohistochemical study in the dog. J. Neurosurg., 66: 741-7. DOI: $10.3171 /$ jns.1987.66.5.0741

Woitzik, J., J.P. Dreier, N. Hecht, I. Fiss and N. Sandow et al., 2012. Delayed cerebral ischemia and spreading depolarization in absence of angiographic vasospasm after subarachnoid hemorrhage. J. Cereb Blood Flow Metab., 32: 203-12. DOI: $10.1038 / j \mathrm{cbfm} .2011 .169$ 